\title{
Relationships between fatty acid status of sow plasma and that of umbilical cord, plasma and tissues of newborn piglets when sows were fed on diets containing tuna oil or soyabean oil in late pregnancy
}

\author{
J. A. Rooke*, I. M. Bland and S. A. Edwards $\dagger$ \\ Animal Biology Division, SAC, Craibstone Estate, Aberdeen AB21 9YA, UK
}

(Received 19 October 1998 - Revised 15 March 1999 - Accepted 23 April 1999)

\begin{abstract}
To investigate the relationships between maternal, umbilical cord and piglet fatty acid status, multiparous sows (six per diet) were fed on diets containing supplements $(30 \mathrm{~g} / \mathrm{kg})$ of either soyabean oil or tuna oil for the last $21 \mathrm{~d}$ of pregnancy. The proportions of most fatty acids differed between diets: in particular, the tuna-oil-containing diet supplied more 22:6n-3 and less 18:2n-6 fatty acids than the soyabean-oil-containing diet. Maternal plasma fatty acid concentrations (mg/l) were greater than those in umbilical plasma and 20:4n-6 and 22:6n-3 fatty acids were present in higher proportions $(\mathrm{g} / 100 \mathrm{~g}$ fatty acids) in umbilical than maternal plasma. Feeding tuna oil increased the proportionate amounts (g/100 g fatty acids) of total $n-3$ fatty acids (particularly 22:6n-3) in umbilical cord, plasma and piglet tissues compared with feeding soyabean oil: in contrast, the proportion of 20:4n-6 was decreased by feeding tuna oil. Changes in piglet fatty acid proportions as a result of oil feeding were not influenced by piglet weight. While proportions of the long-chain $n-3$ and $n-6$ polyunsaturated fatty acids in piglet liver, spleen and reproductive tract (ovaries plus uterus of the female, testes of the male) correlated well with those of umbilical plasma, those in brain and retina were poorly correlated. Therefore umbilical plasma cannot be used to predict the fatty acid status of piglet brain.
\end{abstract}

Pregnancy: Dietary fatty acids: Tissue fatty acids: Pig

Brain and retina contain large proportions of long-chain polyunsaturated fatty acids of the $n-3$ and $n-6$ series, particularly 20:4n-6 and 22:6n-3 (Innis, 1992). In both man and the pig, the period of rapid brain development occurs during the last third of pregnancy and early in postnatal life (Passingham, 1985). An inadequate supply of the appropriate fatty acids, particularly $22: 6 n-3$, has been implicated in impaired visual and cognitive development in man and experimental animals (Uauy et al. 1992; Wainwright, 1992) which may have consequences long into post-natal life (Burdge, 1998). Therefore it is important that an optimal supply of the appropriate fatty acids is available during gestation.

In a previous study (Rooke et al. 1998) the effect on piglet tissue composition and viability of feeding pregnant sows on a diet containing 22:6n-3 in late pregnancy was studied. Concentrations of long-chain $n-3$ polyunsaturated fatty acids in piglet tissues were increased as a result of feeding the sow 22:6n-3 as tuna oil but improvements in piglet viability could not be detected with the number of animals used. To use sufficient animals to determine effects of feeding fatty acids on piglet viability, it will be necessary to have a simple, non-invasive means of monitoring the fatty acid status of the piglet. In man, the fatty acid composition of umbilical plasma and cord tissue has been used to monitor fetal fatty acid status (Crawford et al. 1990), although the use of such data to predict fatty acid status of tissues such as brain has been questioned (Innis, 1992). It was, therefore, the objective of the present study to change the $n-3$ fatty acid status of the pregnant sow in order to quantify relationships between sow fatty acid status and the composition of umbilical cord, plasma and newborn piglet tissue fatty acids. In addition to the tissues sampled previously (liver, brain and retina; Rooke et al. 1998) the fatty acid compositions of entire reproductive tracts and spleens of newborn piglets were also measured. Reproductive tracts were analysed because large amounts of 22:6n-3 are found in the semen of the sexually mature boar, boar spermatozoa phospholipids containing $23-43 \mathrm{~g} / 100 \mathrm{~g}$ fatty acids as 22:6n-3 (Johnson et al. 1972; Evans \& Setchell, 1979; Paulenz et al. 1995). It was of interest, therefore, to determine whether the sexually immature tissues of the newborn piglet differed in their fatty acid composition and in their response to maternal supplementation. Spleen

\footnotetext{
* Corresponding author: Dr John Rooke, fax +44 (0)1224 711292, email j.rooke@ab.sac.ac.uk

$\dagger$ Present address: Department of Agriculture, University of Aberdeen, 581 King Street, Aberdeen AB24 5UA, UK.
} 
was analysed because supplementation of the sow with fish oil during pregnancy and lactation has been shown to alter piglet spleen fatty acid composition and eicosanoid production (Fritsche et al. 1993).

\section{Materials and methods}

\section{Animals}

Twelve pregnant multiparous sows (Large White $\times$ Landrace, Newsham Hybrid Pigs Ltd, Malton, North Yorkshire, UK) were used in the experiment. At the start of the experiment the sows weighed $265 \mathrm{~kg}$, had a mean backfat thickness of $27 \mathrm{~mm}$ at the $\mathrm{P} 2$ position and mean parity was 3.9. Before allocation to diets the sows had been mated with Large White boars, housed in straw-bedded accommodation and fed with $2.5 \mathrm{~kg}$ of a standard commercial pregnancy diet daily. At the start of the experiment, the sows were between 90 and $94 \mathrm{~d}$ of gestation, calculated from the date of first service. The experiment was conducted in September and October 1996.

\section{Experimental design and diets}

The sows were allocated to experimental diets, as they became available, over a period of $14 \mathrm{~d}$ and entered the experiment in two groups of six animals. Within each group, three sows were allocated to a diet containing soyabean oil and the other three sows to a diet containing tuna oil.

Soyabean or tuna oil $(30 \mathrm{~g} / \mathrm{kg}$ diet $)$ was added to a basal diet containing $(\mathrm{g} / \mathrm{kg})$ : milled barley 600 , wheatfeed 188 , soyabean meal 135, fishmeal 52 and mineral and vitamin mix 25 (Sowvite 110; Norvite Ltd, Insch, Aberdeenshire, UK). The mineral and vitamin mix was fortified with $\alpha$-tocopherol acetate as an antioxidant. The expected nutrient analysis of the diet was ( $/ \mathrm{kg}$ fresh weight): $13.5 \mathrm{MJ}$ digestible energy, $180 \mathrm{~g}$ crude protein, $0.94 \mathrm{~g}$ lysine, $59 \mathrm{~g}$ oil and $200 \mathrm{mg} \alpha$-tocopherol acetate. Soyabean oil was obtained from Harbro Farm Sales, Turriff, Aberdeenshire, UK and the tuna oil, which contained a high concentration of 22:6n-3, was Tuna Orbital Oil (Scotia Pharmaceuticals Ltd, Carlisle, Cumbria, UK). Batches of feed were prepared twice weekly by mixing the oil with the basal diet in a $50 \mathrm{~kg}$ capacity horizontal mixer. The feed was stored at ambient temperature in sealed plastic bags with air excluded. Feed samples were obtained when each batch of feed was prepared, and stored at $-20^{\circ}$ to await analysis. Sows were given $2.5 \mathrm{~kg}$ feed daily until parturition and then were transferred to a standard commercial diet.

\section{Experimental procedures}

Sows. Two blood samples were obtained from each sow by jugular venepuncture into evacuated tubes containing potassium EDTA $(18 \mathrm{mg})$, once when allocated to the diets, and again $1 \mathrm{~d}$ before farrowing. Plasma was prepared from blood samples by centrifugation at $2000 \mathrm{~g}$ for $20 \mathrm{~min}$ and stored at $-20^{\circ}$ until analysed.

The sows were transferred to farrowing crates several days before parturition. On day 113 of pregnancy, the sows were injected intramuscularly with $2 \mathrm{ml}$ of a synthetic prostaglandin analogue (cloprostenol, $92 \mathrm{mg} / \mathrm{ml}$; Planate, Coopers Animal Health, Crewe, Ches., UK) at about 11.00 hours to induce farrowing.

Farrowing and piglets. All farrowings were attended. One sow receiving the soyabean oil-supplemented diet produced only four progeny, significantly fewer than for all other litters. Data were not collected for this sow.

As each piglet was born, samples of umbilical cord (50$100 \mathrm{~mm}$ ) and of blood massaged from the cord into a tube containing potassium EDTA $(18 \mathrm{mg})$ were obtained. Samples were not obtained from all piglets because of rupture of the cord during farrowing or, in the case of blood samples, closure of the umbilical blood vessels. After sampling each piglet was removed from the sow to a heated box. After completion of farrowing (30 min after expulsion of the placenta), the weight and sex of each piglet were recorded. From each litter, piglets were selected on the following basis: three piglets were selected from litters of less than or equal to twelve in size and four from litters of more than twelve. The piglets selected were chosen to represent the average weight of the litter but also to include the lightest piglet in each litter. Piglets were anaesthetized by intraperitoneal injection of sodium pentobarbitone $(200 \mathrm{mg} / \mathrm{ml}$; Euthatal, Rhone Merieux, Harlow, Essex, UK) and then immediately killed by an overdose of sodium pentobarbitone. The brain, liver, spleen, eyes and reproductive tract were removed and weights of liver, brain and spleen recorded. Reproductive tracts were defined as ovaries plus uterus in the female and testes in the male. The eyes were freed from connective tissue and the anterior half of the eyeball and contents of the eye removed. The posterior half of the eyeball and liver, brain, spleen and reproductive tract were stored at $-20^{\circ}$ until analysis. Plasma was prepared from the blood samples as described earlier.

Analytical procedures. Feed samples were analysed for $\mathrm{DM}\left(80^{\circ}\right.$ for $\left.18 \mathrm{~h}\right)$, ash $\left(550^{\circ}\right.$ for $\left.18 \mathrm{~h}\right)$, crude protein by the Kjeldahl method and acid ether extract and neutraldetergent fibre according to methods of the Ministry of Agriculture, Fisheries and Food (1992). The estimated digestible energy concentrations of the diets were also calculated according to methods of the Ministry of Agriculture, Fisheries and Food (1993).

Portions of feed or tissue samples were homogenized in a suitable excess of chloroform-methanol $(2: 1, \mathrm{v} / \mathrm{v})$ containing pentadecaenoic acid $(15: 0)$ as internal standard, and extracts of total lipid in chloroform were prepared. Samples of the posterior half of the eyeball were vigorously mixed with saline $(9 \mathrm{~g} \mathrm{NaCl} / \mathrm{l})$ to remove the retinal cells from connective tissue before the retinal cells were extracted. Fatty acid methyl esters were prepared by transmethylation by refluxing the lipid in methanol-toluene-sulfuric acid (20:10:1, by vol.) for $30 \mathrm{~min}$. The fatty acid methyl esters were extracted into hexane before chromatography. They were separated by GLC using a capillary column (HP225, $30 \mathrm{~m} \times 0.25 \mathrm{~mm}$, film thickness $0.25 \mu \mathrm{m}$; Hewlett Packard Ltd, Stockport, Ches., UK) in a Phillips PU4500 chromatograph. Peak areas were determined with the aid of a Kontron DS450 Data System (Kontron Instruments, Watford, Herts., UK) and identified and quantified with reference to the internal standard and a known standard (Supelco 37 Component mix; Supelco, Poole, Dorset, UK). The ratio 
20:4n-6/22:6n-3 was calculated for all samples as an index of long-chain polyunsaturated fatty acid status. For piglet tissues, two additional ratios were calculated; 22:5n-6/ 22:4n-6 (Neuringer et al. 1986) and 22:6n-3/22:5n-6 (Reddy et al. 1994).

\section{Statistics}

Data were analysed by ANOVA techniques using Genstat 5 (1987; Lawes Agricultural Trust; Clarendon Press, Oxford, UK). Where sampling was repeated (sow blood samples) or measurements were made on several animals (piglet tissue samples) a split-plot ANOVA was used with sow as the main plot and sampling time or piglet as the split plot. For data where the number of observations varied because of differences in litter size (umbilical cord and plasma samples) or because piglets were not selected on the basis of sex, a split-plot ANOVA was carried out using the REML procedure of Genstat and diet effects assessed from Wald statistics and $\chi^{2}$ tests. The relationships between umbilical cord or cord plasma fatty acid composition and composition of each piglet tissue were analysed using multiple regression analysis. For each tissue terms for the sex or weight of the piglets were included if significant. To avoid type 1 errors arising because of the number of regression analyses performed, only those relationships for which consistently significant relationships were found across tissues are reported.

\section{Results}

\section{Diet}

The diets fed to the sows did not differ in proximate composition (Table 1). As intended, adding either soyabean oil or tuna oil to the basal diet changed the composition of the dietary fatty acids. The most marked differences were increases in the proportions of 20:5n-3 and 22:6n-3 and a decrease in 18:2n-6 (all $P<0.001$ ) when tuna oil was included in the diet. The tuna-oil diet contained more saturated fatty acids $(P<0.001)$ and a lower $n-6: n-3$ fatty acid ratio $(P<0.01)$ than the soyabean-oil diet.

\section{Sow measurements}

There were changes in the concentrations of fatty acids in sow plasma in relation to both sampling time and diet (Table 2). Fatty acid concentrations did not differ between groups at the start of the experiment. Total plasma fatty acids $(\mathrm{mg} / \mathrm{l})$ decreased $(P<0.001)$ between the start of the experiment and $1 \mathrm{~d}$ before farrowing. The concentration of $18: 2 n-6$ decreased and that of 20:5n-3 increased when tuna oil was fed (diet $\times$ time, $P<0.001$ ) but remained unchanged when soyabean oil was fed. Irrespective of the type of oil included in the diet, the concentration of 20:4n-6 decreased and that of 22:6n-3 increased between the start of the experiment and $1 \mathrm{~d}$ before farrowing; for $22: 6 n-3$ the increase was much greater when tuna oil was fed (interaction, $P<0 \cdot 001)$. As a result the ratio 20:4n-6/22:6n-3 declined with time $(P<0.001)$, the decline being more marked when tuna oil was fed (interaction, $P<0.05$ ).

\section{Piglet measurements}

There were no differences, as a result of the oil fed, in litter size or piglet weight $(P>0.05)$. Mean weight of the piglets selected for tissue analysis $(1.15 \mathrm{~kg})$ was less $(P<0.05$; SED $0.056)$ than that of all piglets $(1.19 \mathrm{~kg})$ as a result of including the lightest piglet in each litter in the slaughter group. There were no significant $(P>0.05)$ between-diet differences for piglet organ weights, although progeny of sows receiving tuna oil (27.6) had heavier brains $(\mathrm{g} / \mathrm{kg}$ live weight; SED $2 \cdot 12, n 6$ ) than the progeny of sows fed with soyabean oil $(25 \cdot 3)$.

Table 1. Composition ( $\mathrm{g} / \mathrm{kg} \mathrm{DM}$ ) of diets containing soyabean oil and tuna oil fed to sows

(Mean values with their pooled standard errors for three samples per diet)

\begin{tabular}{|c|c|c|c|}
\hline Diet... & Soyabean & Tuna & SE \\
\hline $\begin{array}{l}\text { DM }(\mathrm{g} / \mathrm{kg}) \\
\text { Ash } \\
\text { Crude protein } \\
\text { Acid ether extract } \\
\text { Neutral-detergent fibre } \\
\text { Digestible energy }(\mathrm{MJ} / \mathrm{kg} \mathrm{DM})\end{array}$ & $\begin{array}{r}875 \\
58 \\
212 \\
76 \\
189 \\
15 \cdot 6\end{array}$ & $\begin{array}{c}875 \\
58 \\
212 \\
77 \\
194 \\
15.7\end{array}$ & $\begin{array}{l}2 \cdot 7 \\
2 \cdot 4 \\
5 \cdot 1 \\
2 \cdot 8 \\
2 \cdot 0 \\
0 \cdot 10\end{array}$ \\
\hline $\begin{array}{l}\text { Fatty acids }(\mathrm{g} / 100 \mathrm{~g} \text { total fatty } \\
\text { Saturated } \\
\text { Monounsaturated }\end{array}$ & $\begin{array}{l}19.0 \\
21.2\end{array}$ & $\begin{array}{l}30 \cdot 7 \\
24 \cdot 1\end{array}$ & $\begin{array}{l}0.45 \\
0.32\end{array}$ \\
\hline $\begin{array}{l}\text { Total } n-6 \\
18: 2 n-6 \\
20: 4 n-6\end{array}$ & $\begin{array}{l}52 \cdot 5 \\
51 \cdot 7 \\
\text { ND }\end{array}$ & $\begin{array}{r}24.1 \\
23 \cdot 2 \\
1.2\end{array}$ & $\begin{array}{l}0.48 \\
0.54 \\
-\end{array}$ \\
\hline $\begin{array}{l}\text { Total } n-3 \\
18: 3 n-3 \\
20: 5 n-3 \\
22: 5 n-3 \\
22: 6 n-3\end{array}$ & $\begin{array}{l}7 \cdot 4 \\
6 \cdot 9 \\
0.5 \\
\text { ND } \\
\text { ND }\end{array}$ & $\begin{array}{r}19 \cdot 1 \\
3.2 \\
3.9 \\
0.4 \\
11 \cdot 6\end{array}$ & $\begin{array}{l}0.35 \\
0.07 \\
0.07 \\
0.02 \\
0.31\end{array}$ \\
\hline$n-6: n-3$ & $7 \cdot 1$ & 1.4 & 0.06 \\
\hline
\end{tabular}

$\mathrm{ND}$, not detected. 
Table 2. Fatty acid concentrations ( $\mathrm{mg} / \mathrm{l}$ plasma) in plasma obtained from sows before feeding diets containing soyabean oil or tuna oil and after $21 \mathrm{~d}$ feeding in late gestation

(Mean values with their pooled standard errors for six samples per diet)

\begin{tabular}{|c|c|c|c|c|c|c|c|c|}
\hline \multirow[b]{2}{*}{ Fatty acid } & \multicolumn{3}{|c|}{ Sow plasma } & \multirow[b]{2}{*}{ SE† } & \multirow[b]{2}{*}{ SE‡ } & \multicolumn{3}{|c|}{ Statistical significance of effect of: } \\
\hline & Before & Soyabean & Tuna & & & Diet & Time & Diet $\times$ Time \\
\hline Total & 2432 & 2076 & 1659 & 263.2 & $122 \cdot 5$ & NS & $* * \star$ & NS \\
\hline Saturated & 639 & 503 & 452 & $66 \cdot 1$ & $24 \cdot 7$ & NS & *** & NS \\
\hline Monounsaturated & 718 & 455 & 380 & $87 \cdot 8$ & $30 \cdot 0$ & NS & *** & NS \\
\hline$n-3$ & 135 & 164 & 321 & 33.5 & $14 \cdot 3$ & NS & $* * *$ & $* * *$ \\
\hline $18: 3 n-3$ & 52 & 60 & 23 & $12 \cdot 2$ & 8.4 & NS & NS & NS \\
\hline $20: 5 n-3$ & 42 & 45 & 137 & 11.5 & $6 \cdot 0$ & $* *$ & $* * *$ & $* * *$ \\
\hline $22: 5 n-3$ & 10 & 16 & 16 & $5 \cdot 3$ & 2.8 & NS & NS & NS \\
\hline $22: 6 n-3$ & 29 & 44 & 146 & $11 \cdot 6$ & $6 \cdot 1$ & ** & $* * *$ & $* * *$ \\
\hline $20: 4 n-6 / 22: 6 n-3$ & 3.6 & $2 \cdot 1$ & 0.6 & 0.49 & 0.66 & NS & $* * *$ & * \\
\hline
\end{tabular}

${ }^{*} P<0.05,{ }^{* *} P<0.01,{ }^{* * *} P<0.001$.

† Standard error for diet comparisons.

$\ddagger$ Standard error for time $\times$ diet comparisons.

Piglet tissue fatty acid composition. The fatty acid composition $(\mathrm{g} / 100 \mathrm{~g}$ total fatty acids) of both umbilical cord and blood plasma obtained from the cord at birth (Table 3) were influenced by maternal diet. In both sample types, feeding tuna oil increased the proportions of 22:5n-3
$(P<0.01)$ and 22:6n-3 $(P<0.001)$ but decreased the proportion of $20: 4 n-6(P<0 \cdot 001)$. In addition, feeding tuna oil resulted in decreased proportions of $22: 4 n-6$ $(P<0.001)$ in cord and increased proportions of 20:5n-3 $(P<0.001)$ in plasma. As a result the proportion of total

Table 3. Fatty acid composition of umbilical cord ( $\mathrm{g} / 100 \mathrm{~g}$ total fatty acids) and plasma ( $\mathrm{g} / 100 \mathrm{~g}$ total fatty acids and $\mathrm{mg} / \mathrm{l}$ plasma) prepared from umbilical cord blood at birth from piglets born to sows fed on diets containing either soyabean oil or tuna oil from $21 \mathrm{~d}$ pre-partum†

(Mean values for five (soyabean oil) or six (tuna oil) observations and standard errors of the differences between means)

\begin{tabular}{|c|c|c|c|c|c|c|}
\hline & \multicolumn{3}{|c|}{ Cord } & \multicolumn{3}{|c|}{ Plasma } \\
\hline & Soyabean & Tuna & SED & Soyabean & Tuna & SED \\
\hline \multicolumn{7}{|c|}{$\mathrm{g} / 100 \mathrm{~g}$ total fatty acids } \\
\hline Saturated & $44 \cdot 6$ & $44 \cdot 2$ & 0.88 & $42 \cdot 6$ & $41 \cdot 8$ & 1.45 \\
\hline Monounsaturated & $32 \cdot 2$ & $32 \cdot 6$ & 0.72 & $31 \cdot 7$ & $32 \cdot 8$ & 0.82 \\
\hline $\begin{array}{l}n-6 \\
18: 2 n-6 \\
20: 4 n-6 \\
22: 4 n-6\end{array}$ & $\begin{array}{r}17.9 \\
4.0 \\
11.7 \\
1.8\end{array}$ & $\begin{array}{l}14 \cdot 1^{\star \star \star} \\
3 \cdot 5^{\star *} \\
9 \cdot 3^{\star \star *} \\
1 \cdot 1^{\star * \star}\end{array}$ & $\begin{array}{l}1.02 \\
0.67 \\
0.52 \\
0.18\end{array}$ & $\begin{array}{r}18 \cdot 0 \\
7 \cdot 0 \\
9.0 \\
0.6\end{array}$ & $\begin{array}{l}13 \cdot 2^{\star \star \star} \\
5 \cdot 9^{\star \star} \\
5 \cdot 8^{\star \star \star} \\
0 \cdot 5\end{array}$ & $\begin{array}{l}1.20 \\
0.91 \\
0.56 \\
0.17\end{array}$ \\
\hline $\begin{array}{l}n-3 \\
20: 5 n-3 \\
22: 5 n-3 \\
22: 6 n-3\end{array}$ & $\begin{array}{l}3 \cdot 6 \\
1.6 \\
0.9 \\
2 \cdot 4\end{array}$ & $\begin{array}{l}6 \cdot 4^{\star \star \star} \\
1 \cdot 7^{\star \star} \\
1 \cdot 3^{\star \star} \\
3 \cdot 8^{\star \star \star}\end{array}$ & $\begin{array}{l}0.49 \\
0.36 \\
0.18 \\
1.58\end{array}$ & $\begin{array}{l}7 \cdot 6 \\
1.4 \\
0.5 \\
5 \cdot 2\end{array}$ & $\begin{array}{c}12 \cdot 3^{\star \star \star} \\
3 \cdot 7^{\star * \star} \\
0 \cdot 9^{\star *} \\
7 \cdot 8^{\star * \star}\end{array}$ & $\begin{array}{l}0.66 \\
0.35 \\
0.13 \\
0.45\end{array}$ \\
\hline $20: 4 n-6 / 22: 6 n-3$ & $5 \cdot 25$ & $2 \cdot 71^{* \star *}$ & 0.456 & $1 \cdot 75$ & $0 \cdot 80^{* * *}$ & 0.083 \\
\hline $\begin{array}{l}\mathrm{mg} / \mathrm{l} \text { plasma } \\
\text { Total } \\
\text { Saturated } \\
\text { Monounsaturated }\end{array}$ & & & & $\begin{array}{l}510 \\
217 \\
157\end{array}$ & $\begin{array}{l}542 \\
225 \\
178\end{array}$ & $\begin{array}{l}57 \cdot 9 \\
21 \cdot 6 \\
20 \cdot 2\end{array}$ \\
\hline $\begin{array}{l}n-6 \\
18: 2 n-6 \\
20: 4 n-6 \\
22: 4 n-6\end{array}$ & & & & $\begin{array}{r}81 \\
36 \\
30 \\
3\end{array}$ & $\begin{array}{r}73 \\
33 \\
31 \\
3\end{array}$ & $\begin{array}{r}14 \cdot 8 \\
7 \cdot 7 \\
10 \cdot 3 \\
1 \cdot 8\end{array}$ \\
\hline $\begin{array}{l}n-3 \\
20: 5 n-3 \\
22: 5 n-3 \\
22: 6 n-3\end{array}$ & & & & $\begin{array}{r}55 \\
22 \\
2 \\
27\end{array}$ & $\begin{array}{c}67 \\
20 \\
5^{*} \\
40^{\star *}\end{array}$ & $\begin{array}{l}9.9 \\
8.5 \\
1.4 \\
4.0\end{array}$ \\
\hline
\end{tabular}

Mean values were significantly different from those for the corresponding soyabean samples: ${ }^{*} P<0.05,{ }^{* *} P<0.01,{ }^{* * \star} P<0.001$. †For details of diets and procedures, see Table 1 and pp. 214-215. 
Table 4. Fatty acid composition ( $\mathrm{g} / 100 \mathrm{~g}$ total fatty acids) of liver and spleen in piglets born to sows fed on diets containing either soyabean oil or tuna oil from $21 \mathrm{~d}$ pre-partum $\dagger$

(Mean values for five (soyabean oil) or six (tuna oil) observations, with standard errors of the differences between means)

\begin{tabular}{|c|c|c|c|c|c|c|}
\hline \multirow[b]{2}{*}{ Fatty acid } & \multicolumn{3}{|c|}{ Liver } & \multicolumn{3}{|c|}{ Spleen } \\
\hline & Soyabean & Tuna & SED & Soyabean & Tuna & $\overline{\text { SED }}$ \\
\hline $\begin{array}{l}\text { Saturated } \\
\text { Monounsaturated }\end{array}$ & $\begin{array}{l}38.6 \\
31.8\end{array}$ & $\begin{array}{l}39.6 \\
29.8\end{array}$ & $\begin{array}{l}0.94 \\
1.35\end{array}$ & $\begin{array}{l}42 \cdot 7 \\
23 \cdot 4\end{array}$ & $\begin{array}{l}42 \cdot 8 \\
24 \cdot 5^{\star}\end{array}$ & $\begin{array}{l}0.65 \\
0.42\end{array}$ \\
\hline $\begin{array}{l}n-6 \\
18: 2 n-6 \\
20: 4 n-6 \\
22: 4 n-6 \\
22: 5 n-6\end{array}$ & $\begin{array}{r}21.0 \\
6.8 \\
11.5 \\
0.5 \\
0.7\end{array}$ & $\begin{array}{c}15 \cdot 7^{\star \star \star} \\
4 \cdot 7^{\star \star} \\
9 \cdot 0^{\star \star} \\
0 \cdot 3^{\star} \\
0 \cdot 6^{\star}\end{array}$ & $\begin{array}{l}1.03 \\
0.46 \\
0.69 \\
0.06 \\
0.05\end{array}$ & $\begin{array}{r}27 \cdot 9 \\
5 \cdot 6 \\
16 \cdot 7 \\
2 \cdot 2 \\
0.9\end{array}$ & $\begin{array}{c}22 \cdot 9^{\star \star \star} \\
4 \cdot 9^{\star \star \star} \\
14 \cdot 1^{\star \star \star} \\
1 \cdot 3^{\star \star \star} \\
0 \cdot 7^{\star \star}\end{array}$ & $\begin{array}{l}0.68 \\
0.49 \\
0.58 \\
0.15 \\
0.06\end{array}$ \\
\hline $\begin{array}{l}n-3 \\
20: 5 n-3 \\
22: 5 n-3 \\
22: 6 n-3\end{array}$ & $\begin{array}{l}8.6 \\
1.2 \\
0.7 \\
6.4\end{array}$ & $\begin{array}{c}14 \cdot 9^{\star \star *} \\
3 \cdot 6^{\star \star *} \\
1 \cdot 2^{\star} \\
9 \cdot 9^{\star * *}\end{array}$ & $\begin{array}{l}0.76 \\
0.22 \\
0.19 \\
0.54\end{array}$ & $\begin{array}{l}6 \cdot 2 \\
0 \cdot 6 \\
1.4 \\
4 \cdot 0\end{array}$ & $\begin{array}{l}9 \cdot 8^{\star \star \star} \\
2 \cdot 2^{\star \star \star} \\
2 \cdot 0^{\star \star \star} \\
5 \cdot 4^{\star \star \star}\end{array}$ & $\begin{array}{l}0.32 \\
0.08 \\
0.10 \\
0.29\end{array}$ \\
\hline $\begin{array}{l}20: 4 n-6 / 22: 6 n-3 \\
22: 4 n-6 / 22: 5 n-6 \\
22: 6 n-3 / 22: 5 n-6\end{array}$ & $\begin{array}{l}1 \cdot 80 \\
1.75 \\
8 \cdot 86\end{array}$ & $\begin{array}{l}0.92^{\star \star \star} \\
2.02 \\
16 \cdot 82^{\star \star *}\end{array}$ & $\begin{array}{l}0.067 \\
0.186 \\
1.21\end{array}$ & $\begin{array}{l}4.30 \\
0.43 \\
4.25\end{array}$ & $\begin{array}{l}2 \cdot 60^{\star \star \star} \\
0.53^{\star *} \\
7.77^{\star \star \star}\end{array}$ & $\begin{array}{l}0.164 \\
0.029 \\
0.427\end{array}$ \\
\hline
\end{tabular}

Mean values were significantly different from those for the corresponding soyabean samples: ${ }^{*} P<0.05,{ }^{* *} P<0.01$, ${ }^{* * *} P<0.001$.

†For details of diets and procedures, see Table 1 and pp. 214-215.

n-6 fatty acids decreased, $n-3$ fatty acids increased and the ratio 20:4n-6/22:6n-3 decreased (all $P<0 \cdot 001$ ) when tuna oil was fed to the sows. There were significant differences $(P<0.001)$ between the fatty acid composition of cord and that of cord plasma for all fatty acids except for total saturated and $n-6$ fatty acids. The absolute concentrations of fatty acids in umbilical plasma are also given in Table 3 . There were no differences $(P>$ $0.05)$ as a result of maternal diet in total concentrations of fatty acids in umbilical plasma; differences between diets were therefore similar to those described for fatty acids when expressed as a proportion of total fatty acids, although only for 22:5n-3 $(P<0.05)$ and 22:6n-3 $(P<0.01)$ were the differences in concentration significant.

The fatty acid compositions of the piglet tissues sampled at slaughter are shown in Tables 4-6. Piglet weight had no significant effect upon tissue fatty acid composition. However, consistent differences were observed as a result of feeding the two different oils to the sows. The proportions of the long-chain $n-3$ fatty acids, 20:5n-3 and 22:6n-3 were increased in the progeny of sows given tuna oil. These differences were significant for liver $(P<0 \cdot 001)$, spleen $(P<0.001)$, brain $(20: 5 n-3, P<0.05 ; 22: 6 n-3, P<0.01)$ and retina $(20: 5 n-3, P<0.001 ; 22: 6 n-3, P>0.05)$. The

Table 5. Fatty acid composition ( $\mathrm{g} / 100 \mathrm{~g}$ total fatty acids) of brain and retina from piglets born to sows fed on diets containing either soyabean oil or tuna oil from $21 \mathrm{~d}$ pre-partum $\dagger$

(Mean values for five (soyabean oil) or six (tuna oil) piglets, with standard errors of the differences between means)

\begin{tabular}{|c|c|c|c|c|c|c|}
\hline \multirow[b]{2}{*}{ Fatty acid } & \multicolumn{3}{|c|}{ Brain } & \multicolumn{3}{|c|}{ Retina } \\
\hline & Soyabean & Tuna & SED & Soyabean & Tuna & SED \\
\hline $\begin{array}{l}\text { Saturated } \\
\text { Monounsaturated }\end{array}$ & $\begin{array}{l}47 \cdot 7 \\
19 \cdot 6\end{array}$ & $\begin{array}{l}46 \cdot 0 \\
21 \cdot 1\end{array}$ & $\begin{array}{l}1.66 \\
1.38\end{array}$ & $\begin{array}{l}44.5 \\
23.3\end{array}$ & $\begin{array}{l}44.9 \\
23.0\end{array}$ & $\begin{array}{l}0.75 \\
0.76\end{array}$ \\
\hline $\begin{array}{l}n-6 \\
18: 2 n-6 \\
20: 4 n-6 \\
22: 4 n-6 \\
22: 5 n-6\end{array}$ & $\begin{array}{r}18.8 \\
0.5 \\
11.7 \\
4.4 \\
1.4\end{array}$ & $\begin{array}{c}16 \cdot 9^{\star \star} \\
0 \cdot 4^{-10} \\
10 \cdot 9^{\star \star} \\
3 \cdot 7^{\star \star \star} \\
1 \cdot 2\end{array}$ & $\begin{array}{l}0.31 \\
0.06 \\
0.21 \\
0.15 \\
0.13\end{array}$ & $\begin{array}{r}19 \cdot 6 \\
3 \cdot 3 \\
12.4 \\
2.0 \\
0.7\end{array}$ & $\begin{array}{c}16 \cdot 4^{\star \star \star} \\
2 \cdot 4^{\star} \\
11 \cdot 1^{\star \star \star} \\
1 \cdot 5^{\star \star \star} \\
0 \cdot 5^{\star \star}\end{array}$ & $\begin{array}{l}0.32 \\
0.28 \\
0.27 \\
0.04 \\
0.06\end{array}$ \\
\hline $\begin{array}{l}n-3 \\
20: 5 n-3 \\
22: 5 n-3 \\
22: 6 n-3\end{array}$ & $\begin{array}{r}13 \cdot 8 \\
0 \cdot 1 \\
0.5 \\
13 \cdot 3\end{array}$ & $\begin{array}{c}16 \cdot 0^{\star} \\
0 \cdot 2^{\star} \\
1 \cdot 0^{\star \star *} \\
14 \cdot 8^{* \star}\end{array}$ & $\begin{array}{l}0.51 \\
0.05 \\
0.05 \\
0.48\end{array}$ & $\begin{array}{r}12 \cdot 5 \\
0.4 \\
1 \cdot 1 \\
11 \cdot 0\end{array}$ & $\begin{array}{c}15 \cdot 7^{\star} \\
1 \cdot 2^{\star \star \star} \\
1 \cdot 6^{\star \star \star} \\
12 \cdot 8\end{array}$ & $\begin{array}{l}0.97 \\
0.06 \\
0.08 \\
0.95\end{array}$ \\
\hline $\begin{array}{l}20: 4 n-6 / 22: 6 n-3 \\
22: 4 n-6 / 22: 5 n-6 \\
22: 6 n-3 / 22: 5 n-6\end{array}$ & $\begin{array}{l}0.89 \\
0.32 \\
9.8\end{array}$ & $\begin{array}{l}0.74^{\star * *} \\
0.33 \\
14 \cdot 1^{*}\end{array}$ & $\begin{array}{l}0.023 \\
0.004 \\
1 \cdot 19\end{array}$ & $\begin{array}{l}1.17 \\
0.37 \\
15 \cdot 8\end{array}$ & $\begin{array}{l}0.89^{\star *} \\
0.34 \\
27 \cdot 3^{\star \star}\end{array}$ & $\begin{array}{l}0.071 \\
0.037 \\
3 \cdot 28\end{array}$ \\
\hline
\end{tabular}

Mean values were significantly different from those for the corresponding soyabean samples: ${ }^{\star} P<0.05,{ }^{* \star} P<0.01$, ${ }_{\star \star * *} P<0.001$.

†For details of diets and procedures, see Table 1 and pp. 214-215. 
Table 6. Fatty acid composition ( $\mathrm{g} / 100 \mathrm{~g}$ total fatty acids) of ovaries plus uterus from female piglets and testes from male piglets born to sows fed on diets containing either soyabean oil or tuna oil from $21 \mathrm{~d}$ pre-partum†

(Mean values for five (soyabean oil) or six (tuna oil) observations, with the standard errors of differences between means)

\begin{tabular}{|c|c|c|c|c|c|c|c|c|}
\hline \multirow[b]{2}{*}{ Fatty acid } & \multicolumn{2}{|c|}{ Soyabean } & \multicolumn{2}{|c|}{ Tuna } & \multirow[b]{2}{*}{ SED } & \multicolumn{3}{|c|}{$\begin{array}{l}\text { Statistical significance of } \\
\text { effect of: }\end{array}$} \\
\hline & Male & $\overline{\text { Female }}$ & Male & $\overline{\text { Female }}$ & & Diet & Sex & $\overline{\operatorname{Diet} \times \operatorname{Sex}}$ \\
\hline $\begin{array}{l}\text { Saturated } \\
\text { Monounsaturated }\end{array}$ & $\begin{array}{l}45 \cdot 6 \\
25 \cdot 2\end{array}$ & $\begin{array}{l}45 \cdot 5 \\
26 \cdot 7\end{array}$ & $\begin{array}{l}45 \cdot 0 \\
24 \cdot 6\end{array}$ & $\begin{array}{l}45 \cdot 0 \\
26 \cdot 9\end{array}$ & $\begin{array}{l}1.00 \\
0.72\end{array}$ & $\begin{array}{l}\text { NS } \\
\text { NS }\end{array}$ & $\underset{* * *}{N S}$ & $\begin{array}{l}\text { NS } \\
\text { NS }\end{array}$ \\
\hline $\begin{array}{l}n-6 \\
18: 2 n-6 \\
20: 4 n-6 \\
22: 4 n-6 \\
22: 5 n-6\end{array}$ & $\begin{array}{r}22.9 \\
4.2 \\
13.7 \\
2.5 \\
0.7\end{array}$ & $\begin{array}{r}22 \cdot 6 \\
3 \cdot 4 \\
13 \cdot 4 \\
3.4 \\
0 \cdot 6\end{array}$ & $\begin{array}{r}19.3 \\
3.4 \\
12.0 \\
1.7 \\
0.6\end{array}$ & $\begin{array}{r}19 \cdot 6 \\
2.6 \\
12 \cdot 2 \\
2.6 \\
0.5\end{array}$ & $\begin{array}{l}0.94 \\
0.34 \\
0.78 \\
0.20 \\
0.061\end{array}$ & $\begin{array}{c}* * * \\
* \\
* \\
* * * \\
N S\end{array}$ & $\begin{array}{l}\text { NS } \\
\star * * \\
N S \\
\text { NS } \\
\text { NS }\end{array}$ & $\begin{array}{l}\text { NS } \\
\text { NS } \\
\text { NS } \\
\text { NS } \\
\text { NS }\end{array}$ \\
\hline $\begin{array}{l}n-3 \\
20: 5 n-3 \\
22: 5 n-3 \\
22: 6 n-3\end{array}$ & $\begin{array}{l}6 \cdot 3 \\
0.6 \\
1.1 \\
4.5\end{array}$ & $\begin{array}{l}5 \cdot 3 \\
0 \cdot 3 \\
1.2 \\
3 \cdot 7\end{array}$ & $\begin{array}{r}11 \cdot 1 \\
1.7 \\
2.0 \\
7 \cdot 2\end{array}$ & $\begin{array}{l}8 \cdot 6 \\
1 \cdot 1 \\
2 \cdot 2 \\
5 \cdot 3\end{array}$ & $\begin{array}{l}0.62 \\
0 \cdot 15 \\
0.16 \\
0.40\end{array}$ & $\begin{array}{l}* * * \\
\star * * \\
\star * * \\
\star * *\end{array}$ & $\begin{array}{c}* * * \\
* * * \\
* \\
* * *\end{array}$ & $\begin{array}{l}* \\
* \\
\text { NS }\end{array}$ \\
\hline $\begin{array}{l}20: 4 n-6 / 22: 6 n-3 \\
22: 4 n-6 / 22: 5 n-6 \\
22: 6 n-3 / 22: 5 n-6\end{array}$ & $\begin{array}{l}3.06 \\
0.27 \\
6.94\end{array}$ & $\begin{array}{l}3 \cdot 74 \\
0.18 \\
5 \cdot 86\end{array}$ & $\begin{array}{r}1.70 \\
0.33 \\
12.93\end{array}$ & $\begin{array}{r}2 \cdot 27 \\
0.21 \\
10 \cdot 28\end{array}$ & $\begin{array}{l}0.135 \\
0.029 \\
1.216\end{array}$ & $\begin{array}{c}* * * \\
* \\
* * *\end{array}$ & $\begin{array}{l}* * * \\
* * * \\
* *\end{array}$ & $\begin{array}{l}\text { NS } \\
\text { NS } \\
\text { NS }\end{array}$ \\
\hline
\end{tabular}

${ }^{\star} P<0.05,{ }^{* *} P<0.01,{ }^{* * *} P<0.001$.

†For details of diets and procedures, see Table 1 and pp. 214-215.

magnitude of the increase in 22:6n-3 was more marked in liver and spleen than in brain and retina. As a result of the increase in $n-3$ fatty acid proportions there were significant decreases in all tissues in one or more of the longchain fatty acids 20:4n-6, 22:4n-6 and 22:5n-6. Hence, there were significant decreases $(P<0.001$ except for retina, $P<0.01)$ in the $20: 4 n-6 / 22: 6 n-3$ ratio for all tissues; the response was again most marked in liver and spleen.

There was a differential response to feeding tuna oil when the fatty acid composition of reproductive tracts of the piglets was analysed (Table 6). Ovaries plus uterus from female piglets contained higher proportions $(P<0 \cdot 001)$ of monounsaturated fatty acids and a lower proportion of

Table 7. Coefficients for regression equations relating piglet liver, spleen and reproductive tract fatty acids ( $\mathrm{g} / 100 \mathrm{~g}$ total fatty acids) to the corresponding fatty acid in umbilical cord or plasma obtained from the umbilical cord blood at birth

(Mean values with their standard errors)

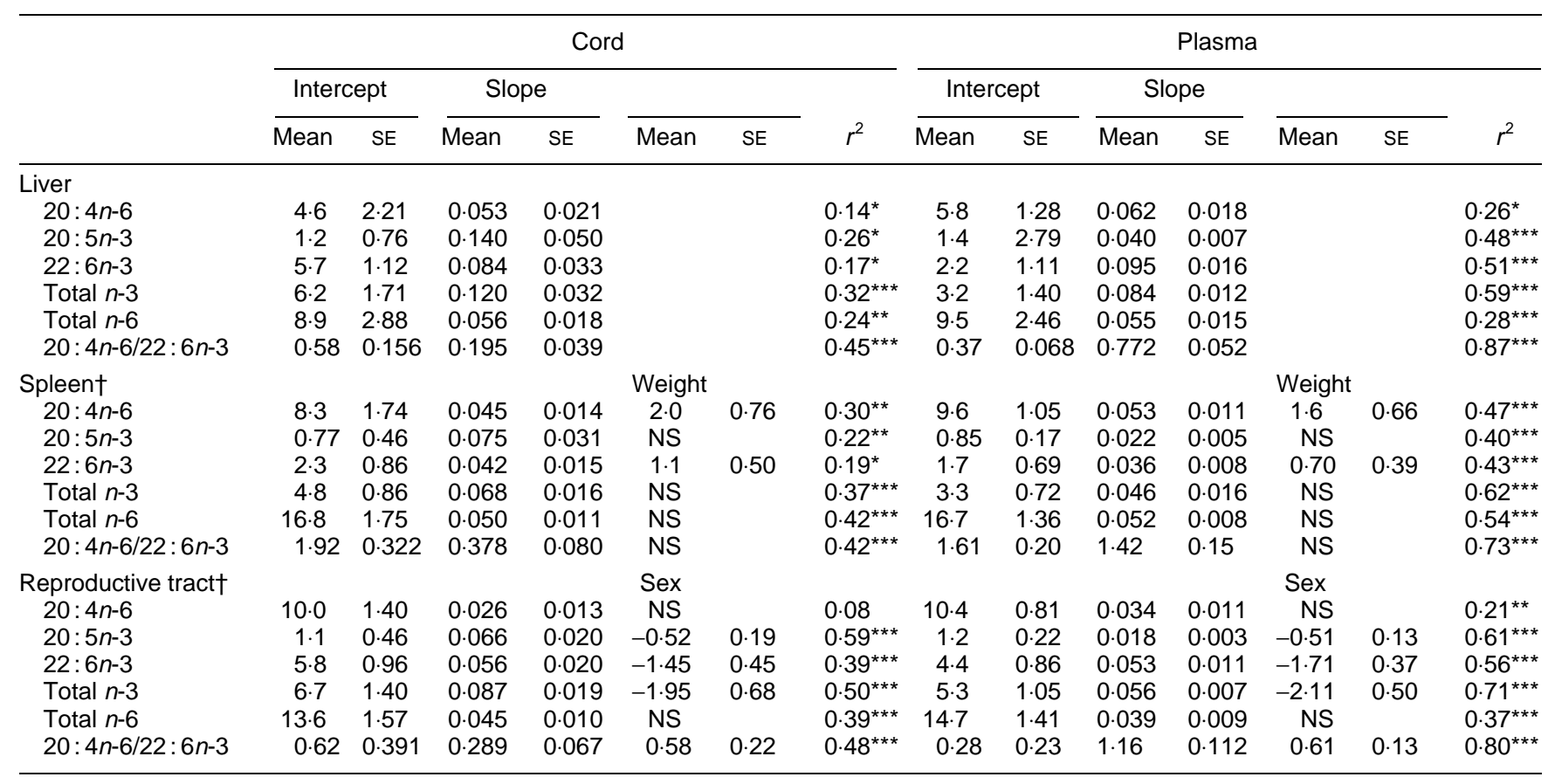

${ }^{*} P<0.05,{ }^{* *} P<0.01,{ }^{* *} P<0.001$.

†For spleen, piglet weight $(\mathrm{kg})$ was included in the regression equation, and for reproductive tract piglet sex (male 1 , female 2$)$ was included in the regression equation. 
Table 8. Coefficients for regression equations relating brain and retina fatty acids $(\mathrm{g} / 100 \mathrm{~g}$ total fatty acids) to the corresponding fatty acid in umbilical cord or plasma obtained from the umbilical cord blood at birth

(Mean values with their standard errors)

\begin{tabular}{|c|c|c|c|c|c|c|c|c|c|c|}
\hline & \multicolumn{5}{|c|}{ Cord } & \multicolumn{5}{|c|}{ Plasma } \\
\hline & Mean & SE & Mean & SE & $r^{2}$ & Mean & SE & Mean & SE & $r^{2}$ \\
\hline \multicolumn{11}{|l|}{ Brain } \\
\hline $20: 4 n-6$ & 9.5 & 0.93 & 0.016 & 0.089 & 0.06 & $10 \cdot 3$ & 0.59 & 0.013 & 0.008 & 0.05 \\
\hline $20: 5 n-3$ & 0.039 & 0.076 & 0.013 & 0.005 & $0.25^{*}$ & 0.11 & 0.028 & 0.003 & 0.007 & $0.35^{\star \star *}$ \\
\hline $22: 6 n-3$ & $12 \cdot 8$ & 0.81 & 0.041 & 0.024 & 0.06 & 1.14 & 0.98 & 0.042 & 0.014 & $0.21^{* *}$ \\
\hline $20: 4 n-6 / 22: 6 n-3$ & 0.65 & 0.037 & 0.041 & 0.009 & $0.38^{\star \star *}$ & 0.66 & 0.034 & 0.116 & 0.026 & $0.36^{* * *}$ \\
\hline \multicolumn{11}{|l|}{ Retina } \\
\hline $20: 4 n-6$ & $10 \cdot 9$ & 0.980 & 0.007 & 0.009 & 0.01 & 9.7 & 5.09 & 0.272 & 0.079 & $0.32^{* * *}$ \\
\hline $20: 5 n-3$ & 0.35 & 0.227 & 0.044 & 0.015 & $0.30^{\star *}$ & 0.53 & 0.095 & 0.011 & 0.003 & $0.37^{* * *}$ \\
\hline $22: 6 n-3$ & $9 \cdot 4$ & 1.45 & 0.083 & 0.043 & 0.08 & 8.2 & 1.86 & 0.057 & 0.027 & $0.12^{*}$ \\
\hline Total $n-3$ & $10 \cdot 4$ & 1.54 & 0.078 & 0.029 & $0.17^{\star}$ & $10 \cdot 5$ & 1.67 & 0.036 & 0.015 & $0.13^{*}$ \\
\hline
\end{tabular}

${ }^{\star} P<0.05,{ }^{\star *} P<0.01,{ }^{* \star *} P<0.001$.

18:2n-6 than testes of the male piglet irrespective of the oil fed to the sow. In common with other tissues, feeding tuna oil increased $n-3$ fatty acids and decreased $n-6$ fatty acids, but for 20:5n-3 and 22:6n-3 the response to feeding tuna oil was more marked in male testes than female ovaries plus uterus (interaction, $P<0.05,20: 5 n-3 ; P<0 \cdot 01,22: 6 n-3$ ).

Adding tuna oil to the diets fed did not decrease the ratio $22: 5 n-6 / 22: 4 n-6$ in any tissue. However, the ratio $22: 6 n-3 /$ $22: 5 n-6$ was significantly greater in all tissues when tuna oil was fed instead of soyabean oil.

Regression coefficients calculated between piglet tissue fatty acid proportions and the corresponding fatty acid in umbilical cord or cord plasma are given in Tables 7 and 8 . Only those fatty acids for which there were consistent relationships $(P<0.01$ or greater) across piglet tissues are reported in Tables 7 and 8 . The summary variables, total $n-3$ and $n-6$ fatty acids and the ratio 20:4n-6/22:6n-3 had greater $r^{2}$ values than individual fatty acids for all tissues. Regression coefficients for liver, spleen and reproductive tract composition and umbilical cord plasma were higher than between the corresponding tissue and umbilical cord. There was little difference between regression coefficients relating cord or cord plasma to piglet brain and retina fatty acid composition and $r^{2}$ values for these two tissues were smaller than those for liver, spleen and reproductive tracts.

\section{Discussion}

The purpose of the present experiment was to establish whether the effects of maternal fatty acid supply on piglet composition at birth could be estimated from the composition of the umbilical cord or of blood obtained from the umbilical cord at birth, thus avoiding the necessity of slaughtering animals. The length of time diets were fed and the composition of the experimental diets were intended to be the same as in a previous experiment (Rooke et al. 1998). As a result the changes in both sow plasma and piglet tissue fatty acid composition when tuna oil was given in each experiment were similar. In both the present experiment and that of Rooke et al. (1998), piglet brain weights ( $\mathrm{g} / \mathrm{kg}$ live weight) increased as a result of tuna-oil feeding, but within individual experiments these differences were not significant. However, when the data from the present experiment and that of Rooke et al. (1998) were combined and analysed, the difference in brain weights between piglets fed on the soyabean and tuna oils became significant (soyabean $v$. tuna; 25.3 v. $26.9 \mathrm{~g} / \mathrm{kg}$ live weight; SED $0.66, n$ 42; $P=$ $0 \cdot 027)$. Thus, not only did feeding tuna oil increase the proportion of 22:6n-3 in total brain fatty acids, brain size was also increased; the functional significance of this increase in relation to piglet viability and growth is being investigated. Compared with the ovaries plus uterus of female piglets, testes of male piglets contained higher proportions of 22:6n-3 and the proportionate increase in $22: 6 n-3$ in response to tuna-oil feeding was greater in male than female piglets. Thus, male piglets in utero displayed the ability to concentrate $22: 6 n-3$; the relevance of this finding to the development of sexual maturity warrants further investigation. In agreement with Fritsche et al. (1993) who fed fish oil to sows during both gestation and lactation, the proportions of $n-3$ fatty acids in spleen were increased by feeding tuna oil in the present experiment. Since Fritsche et al. (1993) demonstrated decreased eicosanoid production in splenocytes from piglets whose dams were being given fish oil in their study and feeding fish oil is associated in animal models with a decrease in the severity of inflammation (Blok et al. 1996), feeding fish oil to sows may protect piglets against undesirable inflammatory responses.

The use of umbilical cord tissue and blood for monitoring the fatty acid status of offspring has been extensively studied in man. Characteristic differences between fatty acid proportions and concentrations in maternal plasma and those in umbilical cord and blood have been observed (e.g. Reddy et al. 1994; Al et al. 1995a,b,c; Otto et al. 1997). In human subjects, amounts of fatty acids in 
maternal plasma phospholipids increased during pregnancy (Al et al., 1995c; Otto et al. 1997) with the absolute amounts of 22:6n-3 increasing by the greatest amount. In the present study, amounts of total plasma fatty acids decreased in the last 3 weeks of gestation from 2432 to $1867 \mathrm{mg} / \mathrm{l}$. This difference between the pig and man may be related to the low-fat, cereal-based diet consumed by the pigs $(76 \mathrm{~g}$ acid ether extract $/ \mathrm{kg}$ ) which supplied a smaller proportion of dietary energy as fat than in a typical human diet, therefore representing a different balance between maternal supply and demand for fatty acids in late pregnancy.

The human studies and the present pig data, however, showed similarities: total fatty acid concentrations were greater in maternal than fetal circulation (Al et al. 1995b; Otto et al. 1997) and the proportionate amounts of fatty acids differed between the two circulations. Changes in the ratio of fatty acid concentrations in umbilical plasma to maternal plasma might give an estimate of the effect of fatty acid supplementation of the sow on piglet demand for fatty acids. Ratios were calculated from the data of $\mathrm{Al}$ et al. (1995b), Van Houwelingen et al. (1995) and Otto et al. (1997) for man and for the present experiment. In both species, the fetal : maternal ratio for total fatty acid concentrations was approximately $0 \cdot 3$. In human subjects, the ratio fetal: maternal 20:4n-6 was greater $(0 \cdot 6-0 \cdot 8)$ than for $22: 6 n-3(0 \cdot 4-0 \cdot 6)$ whereas in the sow fed on soyabean oil the ratio for 22:6n-3 (calculated ratio $>1$ ) was greater and for 20:4n-6 (0.35) less than for human subjects. The effect of adding fish oil to the maternal diet in both human subjects (Van Houwelingen et al. 1995) and pigs in the present study was to decrease the ratio fetal: maternal plasma 22:6n-3. These data suggest that fetal demands may have been met by fish-oil supplementation. Regardless of mechanism, measurement of the ratio fetal: maternal plasma 22:6n-3 over a range of maternal dietary 22:6n-3 intakes may provide a method for estimating the optimum intake of $22: 6 n-3$.

In human studies, relatively high proportionate amounts of Mead acid (20:3n-9) have been observed in umbilical arterial tissue and it has been suggested that $20: 3 n-9$ could be used as an index of fetal fatty acid status since these efferent vessels depend on a supply of fatty acids from umbilical arterial blood (Al et al. 1990; Crawford et al. 1990). The use of Mead acid has, however, been questioned as the concentrations of Mead acid are independent of birth weight (Reddy et al. 1994) and not responsive to changes in fatty acid supply (Van Houwelingen et al. 1995). In the current experiment, concentrations of $20: 3 n-9$ were less than $0.5 \mathrm{~g} / 100 \mathrm{~g}$ fatty acids in umbilical tissue, less than reported values for both umbilical vein and artery (e.g. Van Houwelingen et al. 1995) and were unaffected by the maternal diet and weight of the piglet. This would suggest that in the pig, as suggested by Reddy et al. (1994) and Van Houwelingen et al. (1995), Mead acid concentrations in umbilical tissue are not a useful measure of fatty acid status.

Because a deficiency in $22: 6 n-3$ is accompanied by an increase in the conversion of 22:4n-6 to 22:5n-6 (Neuringer et al. 1986), the ratios 22:5n-6/22:4n-6 (Al et al. 1990) and 22:6n-3/22:5n-6 (Reddy et al. 1994) have been suggested as indices of 22:6n-3 status. In the present study, the ratio 22:5n-6/22:4n-6 in piglet tissues increased rather than decreased when 22:6n-3 supply was increased by feeding the sows tuna oil because $22: 5 n-6$ proportions decreased to a greater extent than the proportion of 22:4n-6. In contrast, the ratio $22: 6 n-3 / 22: 5 n-6$ increased with tuna-oil supplementation as would be expected if 22:5n-6 was synthesized in response to a deficiency of 22:6n-3 and therefore the present ratio would appear in the present study to be a more sensitive index of 22:6n-3 status.

Innis (1992) reviewed the use of plasma and erythrocyte fatty acids as indicators of fatty acid status in organs such as brain and retina and concluded that they were not a specific index of fatty acid status as fatty acids could be used as energy substrates depending on the status of the dam and offspring. In addition, any ability of brain and retina to elongate and desaturate 18:2n-6 and 18:3n-3 would influence the need for long-chain polyunsaturated fatty acids. Subsequently, Rioux et al. (1997) using piglets fed on formula-milks containing a range of fatty acid profiles quantified the relationship between plasma fatty acids and liver and brain fatty acids. These authors found that while liver fatty acid concentrations were reasonably well correlated with plasma fatty acids, brain fatty acids were poorly correlated. One explanation for the lack of correlation of brain fatty acid composition with plasma fatty acids was suggested by Berlin et al. (1998) who found that the fatty acid composition of different areas of the brain of minipigs was predicted with differing accuracy from erythrocyte fatty acids: whilst the fatty acid compositions of the caudate nucleus and cerebellum were significantly correlated with erythrocyte fatty acids that of the forebrain was not. Therefore analysing whole-brain fatty acids may mask effects of nutrition on individual regions of the brain. The results of the present experiment in which maternal nutrition was used to manipulate piglet composition tended to agree with those of Rioux et al. (1997) and Berlin et al. (1998). The composition of tissues e.g. liver, spleen and reproductive tracts, which do not contain large proportions of long-chain polyunsaturated fatty acids, correlated better with umbilical plasma fatty acids than those tissues with a high proportion of polyunsaturated fatty acids, e.g. brain and retina, which grow rapidly in late gestation. Therefore umbilical plasma fatty acid proportions were a poor predictor of brain composition in the fetal piglet. A similar pattern was observed when umbilical cord tissue fatty acid concentrations were used to predict piglet tissue fatty acids although the difference between liver, spleen and reproductive tracts on the one hand and brain and retina on the other was much less marked. The inferior relationships observed with umbilical cord tissue can be explained if umbilical plasma fatty acid concentrations reflect shorter-term changes in fatty acid supply than cord tissue whose fatty acid profile may have been influenced by nutrition before the beginning of the experiment.

In conclusion, changes in umbilical cord tissue and plasma fatty acid concentrations in the piglet at birth reflected changes in the fatty acid profile of diets fed to the pregnant sow but did not correlate well with changes observed in the composition of brain and retina in the newborn piglet. Thus, umbilical cord and plasma fatty acid concentrations cannot be used to predict the effects of maternal diet on piglet brain and retina composition. In 
addition, as no effect of piglet weight was observed on tissue fatty acid composition, it was unlikely that differences in placental size and function between piglets in the same litter influenced fatty acid uptake by the piglet.

\section{Acknowledgements}

We thank Maureen Shanks and Hilary Fraser for the care of the pigs, and Morag Ewen and Ian McKay for technical assistance. SAC receives financial support from the Scottish Office Agricultural, Environment and Fisheries Department. The authors are grateful to Scotia Pharmaceuticals for the gift of the tuna oil.

\section{References}

Al MDM, Badart-Smook A, Van Houwelingen AC, Hasaart THM \& Hornstra G (1995a) Fat intake of women during normal pregnancy: relationship with maternal and neonatal essential fatty acid status. Journal of the American College of Nutrition 15, 49-55.

Al MDM, Hornstra G, Vanderschouw YT, Bulstraramakers MTEW \& Huisjes HJ (1990) Biochemical EFA status of mothers and their neonates after normal pregnancy. Early Human Development 24, 239-248.

Al MDM, Van Houwelingen AC, Badart-Smook A, Hasaart THM, Roumen FJ \& Hornstra G (1995b) The essential fatty acid status of mother and child in pregnancy-induced hypertension: a prospective longitudinal study. American Journal of Obstetrics and Gynecology 172, 1605-1614.

Al MDM, Van Houwelingen AC, Kester ADM, Hasaart THM, De Jong AEP \& Hornstra G (1995c) Maternal essential fatty acid patterns during normal pregnancy and their relationship to the neonatal essential fatty acid status. British Journal of Nutrition 74, 55-68.

Berlin E, Bhathena SJ, McClure D \& Peters RC (1998) Dietary Menhaden and corn oils and the red blood cell membrane lipid composition and fluidity in hyper- and normocholesterolemic miniature swine. Journal of Nutrition 128, 1421-1428.

Blok WL, Katan MB \& Van Der Meer JWM (1996) Modulation of inflammation and cytokine production by dietary $(n-3)$ fatty acids. Journal of Nutrition 126, 1515-1533.

Burdge GC (1998) The role of docosahexaenoic acid in brain development and fetal alcohol syndrome. Biochemical Society Transactions 26, 246-251.

Crawford MA, Costeloe K, Doyle W, Leighfield MJ, Lennon EA \& Meadows N (1990) Potential diagnostic value of the umbilical artery as a definition of neural fatty-acid status of the fetus during its growth - the umbilical artery as a diagnostic tool. Biochemical Society Transactions 18, 761-766.

Evans RW \& Setchell BP (1979) Lipid changes in boar spermatozoa during epididymal maturation with some observations on the flow and composition of boar rete testis fluid. Journal of Reproduction and Fertility 57, 189-196.

Fritsche KL, Alexander DW, Cassity NA \& Huang S-C (1993) Maternally-supplied fish oil alters piglet immune cell fatty acid profile and eicosanoid production. Lipids 28, 677-682.

Innis SM (1992) Plasma and red blood cell fatty acid values as indexes of essential fatty acids in the developing organs of infants fed with milk or formulas. Journal of Pediatrics 120, S78-S86.

Johnson LA, Pursel VG \& Gerrits RJ (1972) Total phospholipid and phospholipid fatty acids of ejaculated and epididymal semen and seminal vesicle fluids of boars. Journal of Animal Science 35, 398-403.

Ministry of Agriculture, Fisheries and Food (1992) Analysis of Agricultural Materials, 2nd ed. London: H.M. Stationery Office.

Ministry of Agriculture, Fisheries and Food (1993) Prediction of the Energy Value of Compound Feedingstuffs for Farm Animals. London: MAFF Publications.

Neuringer M, Connor WE, Lin DS, Barstad L \& Luck S (1986) Biochemical and functional effects of prenatal and postnatal $\omega-3$ fatty acid deficiency on retina and brain in rhesus monkeys. Proceedings of the National Academy of Sciences USA 83, 4021-4025.

Otto SJ, Van Houwelingen AC, Antal M, Godfrey K, LopezJaramillo P \& Hornstra G (1997) Maternal and neonatal essential fatty acid status in phospholipids: an international comparative study. European Journal of Clinical Nutrition 51, 232-242.

Passingham RE (1985) Rates of brain development in mammals including man. Brain, Behaviour and Evolution 26, 167-175.

Paulenz H, Taugbøl O, Hofmo PO \& Saarem K (1995) A preliminary study on the effect of dietary supplementation with cod liver oil on the polyunsaturated fatty acid composition of boar semen. Veterinary Research Communications 19, 273-284.

Reddy S, Sanders TAB \& Obeid O (1994) The influence of maternal vegetarian diet on essential fatty acid status of the newborn. European Journal of Clinical Nutrition 48, 358-368.

Rioux FM, Innis SM, Dyer R \& MacKinnon M (1997) Diet-induced changes in liver and bile but not brain fatty acids can be predicted from differences in plasma phospholipid fatty acids in formulaand milk-fed piglets. Journal of Nutrition 127, 370-377.

Rooke JA, Bland IM \& Edwards SA (1998) Effect of feeding tuna oil or soyabean oil as supplements to sows in late pregnancy on piglet tissue composition and viability. British Journal of Nutrition 80, 273-280.

Uauy R, Birch E, Birch D \& Peirano P (1992) Visual and brain function measurements in studies of $n-3$ fatty acids requirements. Journal of Pediatrics 120, S168-S180.

Van Houwelingen AC, Sorensen JD, Hornstra G, Simonis MMG, Boris J, Olsen SF \& Secher NJ (1995) Essential fatty acid status in neonates after fish-oil supplementation during late pregnancy. British Journal of Nutrition 74, 723-731.

Wainwright PE (1992) Do essential fatty acids play a role in brain and behavioral development? Neuroscience and Biobehavioral Reviews 16, 193-205. 